\title{
Enrich multi-channel P2P VoD streaming based on dynamic replication strategy
}

\author{
K.T. Meena Abarna, T. Suresh
}

Department of Computer Science and Engineering, Annamalai University, India

\begin{tabular}{|c|c|}
\hline Article Info & ABSTRACT \\
\hline $\begin{array}{l}\text { Article history: } \\
\text { Received Jun 27, } 2019 \\
\text { Revised Feb 11, } 2020 \\
\text { Accepted Mar 21, } 2020\end{array}$ & $\begin{array}{l}\text { Peer-to-Peer Video-on-Demand }(\mathrm{VoD}) \text { is a favorable solution which } \\
\text { compromises thousands of videos to millions of users with } \\
\text { completeinteractive video watching stream. Most of the profitable P2P } \\
\text { streaming groupsPPLive, PPStream and UUSee have announced a multi- } \\
\text { channel P2P VoD system that approvals user to view extra one channel at } \\
\text { a time. The present multiple channel P2P VoD system resonant a video at }\end{array}$ \\
\hline $\begin{array}{l}\text { Keywords: } \\
\text { Active prediction of dynamic } \\
\text { popularity } \\
\text { Dynamic replication strategy } \\
\text { multi-channel system } \\
\text { P2P }\end{array}$ & $\begin{array}{l}\text { In order to growth the streaming capacity, this paper highlights completely } \\
\text { different effective helpers created resource balancing scheme that actively } \\
\text { recognizes the supply-and-demand inequity in multiple channels. Moreover, } \\
\text { peers in an extra channel help its unused bandwidth resources to peers in } \\
\text { a shortage channel that minimizes the server bandwidth consumption. To } \\
\text { provide a desired replication ratio for optimal caching, it develops a dynamic } \\
\text { replication strategy that optimally tunes the number of replicas based on } \\
\text { dynamic popularity in a distributed and dynamic routine. This work } \\
\text { accurately forecasts the varying popularity over time using Auto-Regressive } \\
\text { Integrated Moving Average (ARIMA) model, an effective time-series } \\
\text { forecasting technique that supports dynamic environment. Experimental } \\
\text { assessment displays that the offered dynamic replication strategy which } \\
\text { should achieves high streaming capacity under reduced server workload } \\
\text { when associated to existing replication algorithms. }\end{array}$ \\
\hline
\end{tabular}

This is an open access article under the CC BY-SA license.

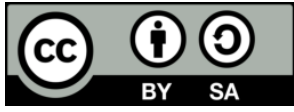

\section{Corresponding Author:}

K.T. Meena Abarna,

Department of Computer Science and Engineering,

Annamalai University,

Annamalai Nagar, Chidambaram, Tamil nadu, India.

Email: abarnakt@yahoo.com

\section{INTRODUCTION}

Problem assertion

$\mathrm{P} 2 \mathrm{P}$ VoD streaming system is totally different from the traditional $\mathrm{P} 2 \mathrm{P}$ sharing networks in various aspects. In P2P VoD system, each video is divided into small chunks those have a critical time constraints. Therefore, it leads a major challenging task to design a chunk scheduling policy for effectively delivering the requested video within its playback deadline. In present multi-channel live P2P video systems, there are numerous performance problems including long playback, long channel switching delay and poor performance for the fewer standard channels.[1] These performance problems are mainly caused due to the two inherent characteristics such as channel dynamic churn and channel resource imbalance. This paper mainly focuses on how to address the constraint by designing effective and optimal replication strategies to improve the streaming capacity with less server bandwidth consumption. First, optimal replication policy is still remains as an open problem so far how to replicate the videos proportional to its popularity. However, It 
results in poor performance especially for unpopular channels and also inaccurate to predict the different viewing popularity over time. Therefore, the main scope of this paper is to propose a time-series analysis techniques to predict the popularity accurately in $\mathrm{P} 2 \mathrm{P}-\mathrm{VoD}$ systems. It achieves an effective replication policy replicate enough replicas based on dynamic popularity, and deficit bandwidth of unpopular movies will contribute the server workload that improves the streaming capacity with reduced server workload.

The main aim and objectives of the paper

To utilize the cross channel peer upload bandwidth for improving the streaming capacity in a multichannel P2P VoD systems.

- $\quad$ To propose an everyday and active replication algorithms in a spread and active fashion.

- $\quad$ To replicate the videos proportional to the dynamic popularity so as to reduce the server workload.

- $\quad$ To propose the peer scheduling policy for effectively deliver the requested video with less intra-domain traffic in a smart and cooperative way.

The main contribution of this paper is

Active Prediction of Dynamic Popularity: This work accurately predicts the dynamic popularity over time using standard linear predictor model called as autoregressive integrated moving average model $\operatorname{ARIMA}(\mathrm{x}, \mathrm{y}, \mathrm{z})$. This model deploys an effective time-series forecasting technique to predict the popularity even under dynamic environment.

- Dynamic Replication based on Optimal Tuning: This work proposes an effective dynamic replication that replicates the video proportionally based on dynamic popularity in a distributed and dynamic manner. In order to optimize the replication in a high dynamic P2P VoD system, this approach proposes two types of tuning such as Proactive and Reactive tuning methods to achieve the desired replication ratio.

\section{RESEARCH METHOD}

The main purpose of this work is to recover the streaming size of a multi-channel P2P video system by irresistible its two fundamental characteristics: channel churn and channel resource imbalance. While regarding the multi-channel systems [2], Wu et. al [3] proposes two strictly linked papers to recover the server bandwidth utilization in multi-channel P2P streaming systems. In [3], Wu et al. proposed, an online server capacity provisioning algorithm to alter the supply of server bandwidth proactively between the dissimilar channels rendering to the expected demand, the streaming quality, and the priorities of channels. In [4], Wu et al.proposed the effective optimization algorithm to reduce the main two problems such as dynamic channel churn and channel resource imbalance between multiple channels. Hence, this method permits active cross channel resource distribution and also achieves reliability in multi-channel P2P streaming systems.

In order to analyze the server bandwidth consumption among the peers with either homogeneous or heterogeneous upload capacity, three models [5-7] are built in multi-channel VoD systems. Kumar et al. [5] develop a simple stochastic fluid model that seeks to expose the performance limitations of channel based P2P streaming systems. This model provides closed-form expressions to examine the fundamental characteristics of a P2P streaming system, including the dynamic churn, heterogeneous upload capacity, limited infrastructure capacity and peer buffering and playback delay. However, this study assumed that channels are of homogeneous playback rates. Moreover in [1], the same authors further extended a tractable infinite server queuing network model to capture heterogeneous peer channel switching patterns. This paper analytically studies the performance of multi-channel systems by considering peer channel switching, peer churn, bandwidth heterogeneity and zipf-like channel popularity. In [2], Linear programming model differs from the aforementioned two models because it is independent of implementations. This approach provides a closed form solutions due to its linear property that paves a way to explore the design space of multichannel systems numerically. In [8], the linear programming model used to analyze the three bandwidth allocation schemes such as Naive Bandwidth allocation Approach (NBA), Passive Channel-aware bandwidth allocation Approach (PCA) and Active Channel-aware bandwidth allocation Approach (ACA). They are analyzed in terms of streaming quality, channel structure, implementation complexity, peer population distributions and upload bandwidth distributions using bandwidth satisfaction ratio. Compared to NBA and PCA designs, ACA design provides effective performance since it allows a peer to subscribe to unwatched channels. However, this approach does not compare the system performance under peer dynamics (channel switching/peer leaving) in the transition state. Still, none of these proposals has taken collaboration of normal peers in multiple $\mathrm{VoD}$ channels into consideration. 


\subsection{System model}

In this work, we consider a system model of multi-channel P2P-VoD system[1] G represent as $\{\mathrm{S}, \mathrm{P}, \mathrm{V}\}$ where $\mathrm{S}$ is the streaming server of the system, $\mathrm{P}$ is a set of peers $\left\{\mathrm{p}_{1}, \mathrm{p}_{2} \ldots \mathrm{p}_{\mathrm{N}}\right\}$ and $\mathrm{V}$ is a set of videos represent as $\left\{\mathrm{v}_{1}, \mathrm{v}_{2} \ldots \mathrm{vk}\right\}$. Peers in the system include both set of active peers $\left\{\mathrm{ps}_{1}, \mathrm{ps}_{2} \ldots \mathrm{ps}_{\mathrm{h}}\right\}$ and set of inactive peers $\left\{p_{\text {rep } 1} p_{\text {rep } 2 \ldots} \ldots p_{\text {repk }}\right\}$. The proposed system encompasses $m$ number of channels $\left\{\operatorname{ch}_{1}, \operatorname{ch} 2 \ldots\right.$ $\left.\mathrm{ch}_{\mathrm{m}}\right\}$ where each channel consist of set of client peers connected by mesh based P2P links $\left\{1_{1}, 12 \ldots 1_{R}\right\}$.

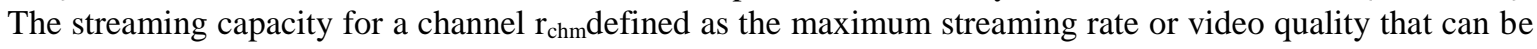
obtained by each peer in the channel $\mathrm{ch}_{\mathrm{m}}$. The server upload bandwidth allocated to the set of channels represented as $\left\{\mathrm{s}_{\mathrm{ch} 1}, \mathrm{~s}_{\mathrm{ch} 2 \ldots} \ldots \mathrm{s}_{\mathrm{chm}}\right\}$. Let $\mathrm{U}_{\mathrm{N}}$ is the maximum upload capacity of peer $\left(\mathrm{p}_{\mathrm{N}}\right)$. In this $\mathrm{P} 2 \mathrm{P}$ assisted peer scheduling policy, a peer wants to view a video $v_{k}$ can access the data from three components such as i) streaming server ii) synchronized (active) peers denotes as $\left\{\mathrm{ps}_{1}, \mathrm{ps}_{2} \ldots \mathrm{ps}_{\mathrm{h}}\right\}$ which are currently watching the video $\left(\mathrm{v}_{\mathrm{k}}\right)$ and iii) replication (inactive) peers denotes as $\left\{\mathrm{p}_{\text {rep } 1} \mathrm{p}_{\text {rep } 2 \ldots} \ldots \mathrm{p}_{\text {repk }}\right\}$ which are not currently viewing the video but cached in its local cache space. Each peer downloads the video with a playback rate $\left(\mathrm{p}_{\mathrm{r}}\right)$ to achieve less start-up latency and reliable streaming quality. Each peer independently maintains the local cache to store the cached videos. When a peer enters or leaves the system, video replica is cached or gets ejected; the peer must send heart beat messages to the tracker in order to indicate its active presence in the channel. Therefore, tracker maintains both the peer statistics and cached information about the peers in the network. This proposed approach undergoes two processes such as i) Synchronous uploading: synchronous peer who entered earlier than requesting peer $\left(\mathrm{P}_{\text {req }}\right)$ can able to provide the requested video for $\mathrm{P}_{\text {req. }}$ ii) Greedy scheduling: maximum cooperation between the synchronous peers to maximize the number of peers to download data at the required playback rate with high streaming quality.

\subsection{Dynamic replication based on optimal tuning}

This work proposes an effective dynamic replication [9] that replicates the video proportionally based on deficit bandwidth in a distributed and dynamic manner. Therefore, it minimizes the upload bandwidth consumption of server $\mathrm{S}$ that reduces the server workload due to the caching of enough replicas among the peers in multi-channel $\mathrm{P} 2 \mathrm{P} \mathrm{VoD}$ systems. It provides a low start up latency for accessing the videos and supports a required playback rate to improve the streaming capacity. In order to optimize the replication effectively in a high dynamic P2P VoD system [1], this approach proposes two types of tuning such as Proactive and Reactive tuning methods tune the number of replicas to achieve desired replication ratio [10].

\subsubsection{Reactive tuning}

When the cache of peer is full, then effective cache replacement algorithm tunes the replication ratio according to the deficit bandwidth $\left(\mathrm{U}_{\mathrm{def}}\right)$ in order to reduce the server workload. Peer has to decide the video to be removed from the local cache to provide space for newly arrived data. In that case, peer replaces the cached data item based on its Enough Replica (ER) index that computes from the deficit bandwidth. Cache replacement algorithm is illustrated as follows.

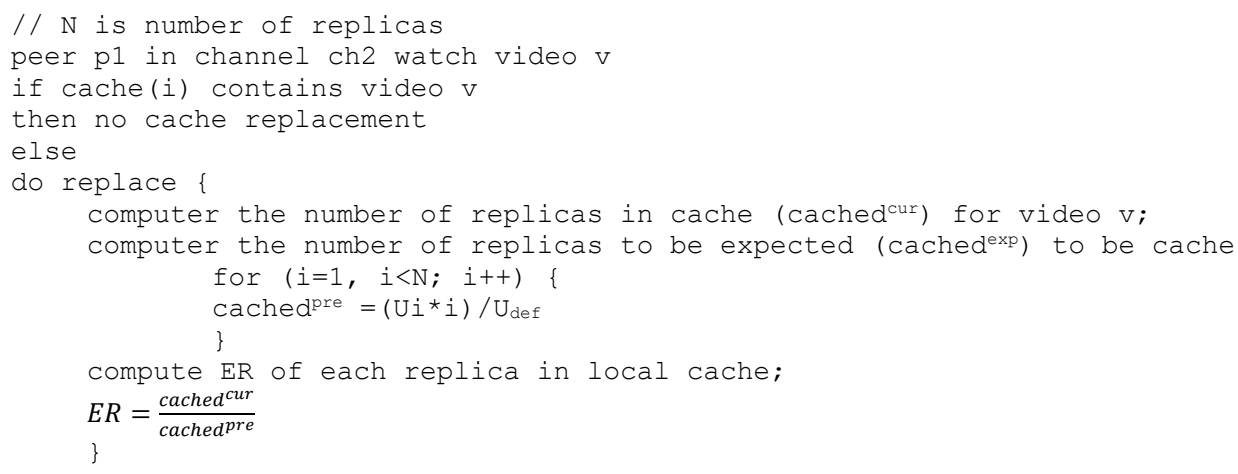

This replacement approach computes the enough replica index to determine that whether replica of the video is enough to meet the future request. The ER index is the ratio of the number of current cached data items (cached $\left.{ }^{\text {cur }}\right)$ and the number of expected data to be cached (cached $\left.{ }^{\text {pre }}\right)$.

$$
E R=\frac{\text { Number of current cached data items }\left(\text { cached }^{\text {cur }}\right)}{\text { Number predicted data items to be cached }\left(\text { cached }^{\text {pre }}\right)}
$$


If the computed ER index is less than one $(\mathrm{ER}<1)$, the number of available replicas is not enough to meet the required replication ratio. On the contrary, computed ER index is greater than one $(\mathrm{ER}>1)$, the number of replicas is much enough to meet the desired replication ratio. Therefore, cached replicas which has high ER index remove out from the cache in order to evict the unwanted replicas when the cache is full. Therefore, enough replica index based cache replacement techniques reduces the server load and also achieves the optimal desired replication ratio.

\subsubsection{Proactive tuning}

Cache replacement algorithm reduces the server workload only in the case when the popularity of the video is uniform all over the time. However, the popularity of a video may varies dynamically (nonstationary), therefore, server workload increases when there is surge demand for video. It degrades the system performance and takes longer time to obtain the desired replication ratio. Therefore, this work proposes a proactive approach that predicts the popularity using standard linear predictor model called as autoregressive integrated moving average model ARIMA (x, y, z) [9]. This model deploys an effective timeseries forecasting technique to predict the popularity even under dynamic environment. In order to predict the popularity, this approach differentiates the non-stationary time series $\left(\mathrm{NS}_{\mathrm{t}}\right)$ into y times for deriving the stationary time series $S_{t}$. The stationary time series $S_{t}$ is the linear weighted sum of $x$ values in the series $\left\{\mathrm{S}_{\mathrm{t}-1}, \mathrm{~S}_{\mathrm{t}-2} \ldots \mathrm{S}_{\mathrm{t}-\mathrm{x}}\right\}$ and $\mathrm{z}$ number of casual errors $\left\{\mathrm{E}_{\mathrm{t}-1} \ldots \mathrm{E}_{\mathrm{t}-\mathrm{z}}\right\}$ This approach deploys two phases such as regression model recognition and regression model evaluation to predict the popularity of the data item linearly. Regression model recognition identifies the appropriate values for $\mathrm{x}, \mathrm{y}$, $\mathrm{z}$. And then, in the regression model evaluation estimates the coefficients for the linear weighted summation that is $\mathrm{x}+\mathrm{Z}$; Thus, the popularity prediction for time $t+1$ computes ARIMA $(0,3,2)$ as follows

$$
\overline{\mathrm{P}_{\mathrm{t}+1}}=3 \mathrm{P}_{\mathrm{t}}-2 \mathrm{P}_{\mathrm{t}-1}+\mathrm{E}_{\mathrm{t}+1}-\beta \mathrm{E}_{\mathrm{t}}
$$

Here, $\beta$ is the co-efficient for the casual error ( $E_{t}$ and then casual error at a future time $E_{t+1}$ is zero and then

$$
E_{t}=\bar{P}_{t}-P_{t}
$$

Substitute $E_{t}$ in equation

$$
\overline{\mathrm{P}_{\mathrm{t}+1}}=3 \mathrm{P}_{\mathrm{t}}-2 \mathrm{P}_{\mathrm{t}-1}-\beta\left(\overline{\mathrm{P}}_{\mathrm{t}}-\mathrm{P}_{\mathrm{t}}\right)
$$

In order to derive the coefficient $\beta$, this approach dynamically refines the ARIMA model by training the model using popularity statistics of the most recent $n$ times in a dynamic fashion and then value of the coefficient of casual error $\beta$ is derived. The popularity of the data is accurately predicted using (2). Dynamic Popularity based Push algorithm are explained as follows

Step 1: Peer statistics Collection

Number of active peers in ech channel

Step 2: Popularity Statistics Collecton

Statistic: collect the most recent $\mathrm{n}$ times popularitu statistics

Step 3: Supervise learning the model

Train ARIMA $(0,3,2)$ using collected popularity statistics

Step 4: Popularity Prediction

Predict the video popularity for time $\mathrm{t}+1$ at time $\mathrm{t}$

$\bar{P}_{(\mathrm{t}+1)}=3 P_{t}-2 P_{t-1} \beta\left(\mathrm{P}_{t}-\mathrm{Pt}\right)$

Step 5: Checking of confidence interval for predicted popularity

if $\left(\bar{P}_{(\mathrm{t}+1)}\right.$ lies with in confifence interval of $\left.P_{t+1}\right)$

Correct Popularity Prediction:

else

retrain the ARIMA $(0,3,2)$ using collected popularity statistics.

Step 6: Push approach to tune replica

if $(\mathrm{P}(\mathrm{v})>$ Threshold $)$

Replicate the video $\mathrm{v}$ to meet desired replication ratio

else

push out the video v; 
In this Active tuning, each peer actively replicates the number of replicas of data items, which have predicted high popularity in the future. On the other hand, less popular data items with predicted low popularity are pushed out when its number of replications is greater than the desired replication ratio. In this proactive tuning approach, each peer proactively predicts the popularity of the data item in its local cache during idle time. Then, each peer replicates the data item based on its predicted popularity in order to meet the desired replication ratios. It reduces the server workload at the same time improves the streaming quality

\section{RESULTS AND ANALYSIS}

This section carry out an extensive simulation to validate and evaluate the performance of proposed dynamic replication strategy with helpers based resource channel allocation approach. Experimental set up comprises a nearly 5,000 peers and streaming server provide nearly 200 videos with an upload capacity of $70 \mathrm{Mbps}$. Each video plays at a playback rate $(\mathrm{Pr})$ of ${ }_{500 \mathrm{Kbps}}$ and its popularity follows Zipf distribution. The size of the video is 60 minutes, which is consistently divided into 60 segments. The peer's upload and download sizes uniformly spread among in the range of $1.5 \mathrm{Mbps}$ and 3.0 Mbps. Each peer preserves a buffer with a capacity of 5 sections. The playback time of each peer is dispersed randomly between 0 and 60 minutes. Moreover, experimental results show the helpfulness of the proposed approach in terms of streaming capacity and reduction of server load by comparing with the existing proportional replication approach.

\subsection{Average streaming capacity}

Figure 1 shows that the proposed dynamic replication approach achieves high streaming capacity than the existing proportional approach in accordance to the increasing server upload capacity. In proposed approach, the streaming capacity increases gradually by optimally allocating the server upload capacity among the multiple channels using helpers based resource sharing scheme. Whereas in the existing scheme, peer bandwidth resources shares within the same channel, not among the multiple channels. Therefore, expected streaming quality cannot be acquired in proportional replication approach.

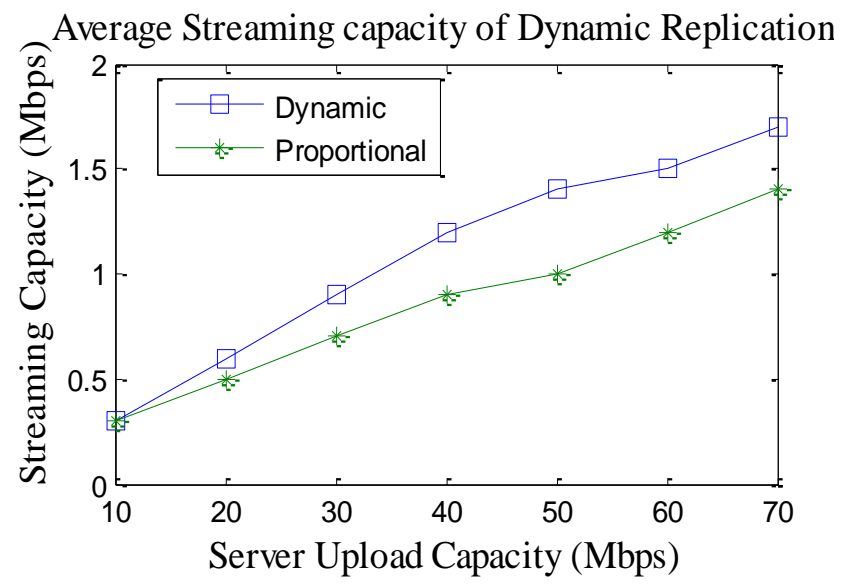

Figure 1. Average streaming capacity in dynamic replication

\subsection{Reduction of server load}

\subsubsection{Effect of cache size}

Figure 2 shows the reduction of server load in a proposed dynamic replication approach. According to the increasing cache size, active peers itself can able to satisfy the request from any other peers in the system. Therefore, requested peer does not depend upon the server for streaming. Thus, the server workload can be predominantly reduced according to the increasing cache size. The following graph clearly examines that when the cache size is small, both the approaches come under the same server workload. In Dynamic Replication, peers replicate videos in a smart and cooperative way to avoid repeatedly caching of videos that are likely to be cached by the other peers in the system. More importantly, when the cache is large enough, proposed dynamic replication performs better than the existing proportional replication approach. 


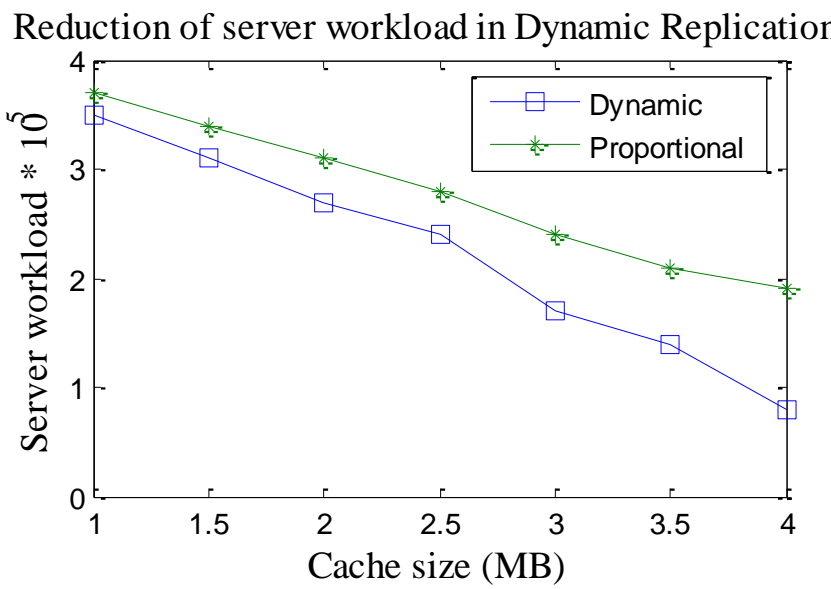

Figure 2. Reduction of server load in dynamic replication

\subsubsection{Effect of the number of peers}

Figure 3 shows that the dynamic replication approach acquires less server workload than the already existing proportional replication approach even under a large number of peers. This is because; proposed approach signifies the effective replication of the number of replicas in a distributed and dynamic manner that makes a better use of the peers' cache space. Therefore, effective maintaining of the videos in a cache space improves the streaming quality and also reduces the server workload.

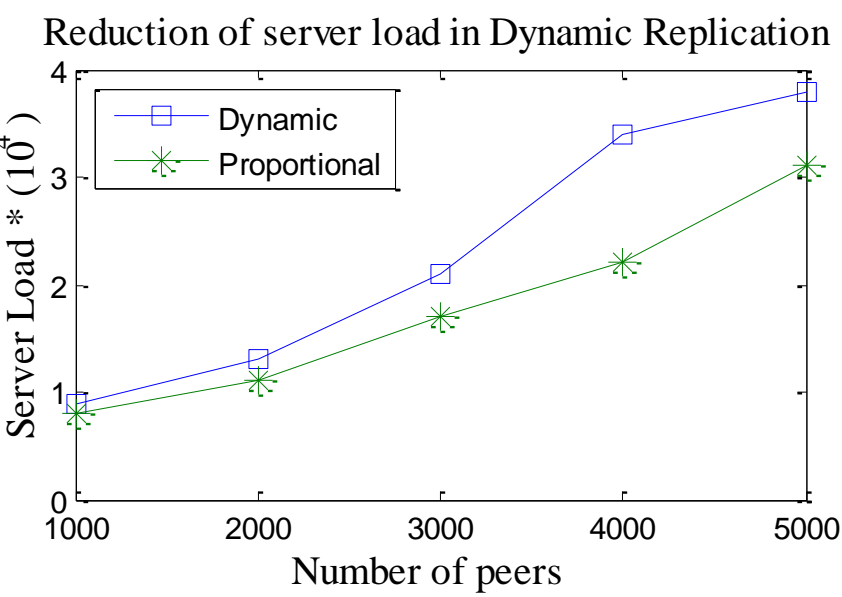

Figure 3. Reduction of server load in dynamic replication

\section{CONCLUSION}

This paper mostly reflects the recently emerging multi-channel $\mathrm{P} 2 \mathrm{P}$ video-on-demand streaming service and the problem of how to use collaboratively. In order to optimize the replication, this paper proposed two types of tuning such as passive and active tuning to achieve desired replication ratio Furthermore, presented a mathematical framework to optimize the replication based on dynamic popularity. The proposed algorithms are effective evenunder a dynamic churn and videos with different viewing popularity. It uses an effective time series forecasting technique, autoregressive integrated moving average model ARIMA (x, y, z) to predict the popularity over time. Experimental evaluation illustrates that proposed approach achieves high streaming capacity and less server workload than existing replication approaches. 


\section{REFERENCES}

[1] Z. Wang, C. Wu, L. Sun, and S. Yang, "Strategies of collaboration in multi-channel P2P VoD streaming," IEEE Global Telecommunications Conference, pp. 1-5, 2010.

[2] Yifeng He and Ling Guan, "Improving streaming capacity in multi-channel P2P VoD systems via intra-channel and cross-channel resource allocation," International Journal of Digital Multimedia Broadcasting, vol. 2012, 2012.

[3] C. Wu, B. Li, and S. Zhao, "Multi-channel live P2P streaming: Refocusing on servers," Proceedings of the IEEE international conference on Computer Communications, 2008.

[4] D. Wu, C. Liang, Y. Liu, and K. Ross, "View-upload decoupling: A redesign of multi-channel P2P video systems," Proceedings of the IEEE 28th Conference on Computer Communications, pp. 2726-2730, Apr 2009.

[5] R. Kumar, Y. Liu, and K. Ross, "Stochastic fluid theory for P2P streaming systems," Proceedings of the $26^{\text {th }}$ IEEE International conference on Computer Communications, pp. 919-927, 2007.

[6] D. Wu, et al., "Queuing network models for multichannel P2P live streaming systems," Proceedings of the IEEE International conference on Computer Communications, pp. 73-81, 2009.

[7] M. Wang, L. Xu, and B. Ramamurthy, "Linear programming models for multi-channel P2P streaming systems," Proceedings of the IEEE INFOCOM, pp. 1-5, 2010.

[8] Miao Wang, et al., "Exploring the design space of multichannel peer-to-peer live video streaming systems", IEEE/ACM Transactions on Networking, vol. 21, no. 1, pp. 162-175, 2013.

[9] Y. Zhou, T.Z.J. Fu, et al., "A unifying model and analysis of P2P VoD replication and scheduling”, Proceedings of the IEEE INFOCOM, pp. 1530 - 1538, 2012.

[10] W. Wu and J. Lui, "Exploring the optimal replication strategy in P2P VoD systems: characterization and evaluation", In Proceedings of the 30th IEEE International Conference on Computer Communications, pp. 1206-1214, 2011. 